\title{
Oblivious Enforcement of Hidden Information Release Policies
}

\author{
Brian Wongchaowart \\ bpw5@cs.pitt.edu \\ Adam J. Lee \\ adamlee@cs.pitt.edu \\ Department of Computer Science \\ University of Pittsburgh \\ 210 S. Bouquet St. \\ Pittsburgh, PA 15260
}

\begin{abstract}
In a computing system, sensitive data must be protected by release policies that determine which principals are authorized to access that data. In some cases, such a release policy could refer to information about the requesting principal that is unavailable to the information provider. Furthermore, the release policy itself may contain sensitive information about the resource that it protects. In this paper we describe a scheme for enforcing information release policies whose satisfaction cannot be verified by the entity holding the protected information, but only by the entity requesting this information. Not only does our scheme prevent the information provider from learning whether the policy was satisfied, but it also hides the information release policy being enforced from the requesting principal. Unlike previous approaches, our construction requires no guesswork or wasted computation on the part of the information requester. The information release policies that we consider can contain third-party assertions that themselves have release conditions that must be satisfied; we show that our system functions correctly even when these dependencies form cycles.
\end{abstract}

\section{Categories and Subject Descriptors}

D.4.6 [Operating Systems]: Security and Protection-access controls, authentication; K.6.5 [Management of Computing and Information Systems]: Security and Protection-authentication

\section{General Terms}

Security

\section{Keywords}

Hidden policies, hidden credentials, distributed proof

Permission to make digital or hard copies of all or part of this work for personal or classroom use is granted without fee provided that copies are not made or distributed for profit or commercial advantage and that copies bear this notice and the full citation on the first page. To copy otherwise, to republish, to post on servers or to redistribute to lists, requires prior specific permission and/or a fee.

ASIACCS'10 April 13-16, 2010, Beijing, China

Copyright 2010 ACM 978-1-60558-936-7 ...\$10.00.

\section{INTRODUCTION}

In this paper we consider an access control setting in which Alice would like to obtain a secret $s$ from Bob, but Bob has a policy requiring that a conjunction of quoted assertions made by other principals must be true before $s$ can be disclosed to Alice. By a "quoted assertion" we mean a proposition of the form $p_{i}$ says $e_{i}$, where $e_{i}$ is an arbitrary expression that can be asserted by some principal $p_{i}$; the quoted assertion $p_{i}$ says $e_{i}$ is true if $p_{i}$ is willing to assert $e_{i}$. For example, $e_{i}$ may be the assertion that Alice has some attribute (e.g., that Alice is a student), and $p_{i}$ may be an authority trusted by Bob to know whether Alice does in fact have the attribute in question (e.g., the registrar at an accredited university).

If Bob is able to determine the truth of each proposition $p_{i}$ says $e_{i}$ in his release policy for $s$, then he can decide whether to release $s$ to Alice without any further interaction with her. But Bob's policy may also refer to private attributes of Alice that he is not authorized to learn about directly from the relevant authority. In this case, Bob could ask Alice to prove that she satisfies his policy by obtaining digitally signed credentials attesting to the truth of each quoted assertion that he cannot evaluate himself. This solution may be unsatisfactory for both parties, however. On the one hand, Bob may have to reveal parts of his policy that he considers sensitive to Alice, as the release policy for a secret can reveal information about the secret itself. On the other, Alice may have to disclose the truth of quoted assertions that she considers private to Bob.

If there were a universally trusted third party (TTP), solving this problem would be simple: Bob discloses his secret $s$ and its release policy $p$ to the TTP, which queries principals about the truth of the quoted assertions in $p$ and releases $s$ to Alice if and only if $p$ is satisfied. Alice learns nothing about Bob's policy aside from whether it was satisfied (unless she is queried by the TTP herself), Bob does not learn whether Alice receives the secret, and no expensive cryptography is needed. In this paper we show that a simple and computationally efficient solution is possible even without a TTP if Bob has a basic level of trust in the principals whose quoted assertions his policy depends on. Specifically, Bob must only trust these principals not to reveal their interactions with Bob to Alice. In our approach, Bob does not have to be able to learn the truth value of a quoted assertion in his policy if Alice is allowed to do so, while Alice learns no more about Bob's policy than she would learn from in- 
teracting with a TTP. Because Bob does not learn whether his policy was satisfied, we say that our protocol permits the oblivious enforcement of a hidden information release policy.

This paper is organized as follows. Section 2 develops the intuition behind our construction, while the full protocol details are given in Section 3. Section 4 presents a sample run. We briefly compare our approach to related work in Section 5, and summarize in Section 6.

\section{PROTOCOL INTUITION}

Our construction relies on a public-key encryption scheme supporting a multiplicative homomorphism. In such an encryption scheme, if $E\left(m_{1}\right)$ and $E\left(m_{2}\right)$ represent the encryptions of messages $m_{1}$ and $m_{2}$ using the same public key, then these two ciphertexts can be combined to yield $E\left(m_{1} \cdot m_{2}\right)$ without knowledge of the private key required to decrypt $E\left(m_{1}\right)$ and $E\left(m_{2}\right)$. We further require that the encryption scheme have the property of IND-CPA security, or indistinguishability of ciphertexts under chosen-plaintext attack, which ensures that no information can be obtained about the plaintext corresponding to a given ciphertext without knowledge of the private key. A concrete example of an encryption scheme with these properties is ElGamal encryption [5] using a group in which the decision Diffie-Hellman (DDH) assumption [1] holds.

Suppose that a principal $p_{0}$ wishes to obtain a secret $s$ from principal $p_{1}$, but that $p_{1}$ is only willing to disclose $s$ to $p_{0}$ if certain conditions - the release policy for $s$-hold. We model $p_{1}$ 's release policy for $s$ as a set of quoted assertions of the form $p_{i}$ says $e_{i}$, where $e_{i}$ is any expression that can be evaluated to a Boolean value by $p_{i}$. If $p_{1}$ 's release policy requires that some of these assertions must be true simultaneously, then each relevant $e_{i}$ should include the constraint that the expression must evaluate to true throughout a time interval long enough to ensure that the expressions are simultaneously true at some point in time despite any clock differences between the principals.

Since $p_{1}$ can trivially enforce the part of a release policy that depends only on information that it can obtain from its local knowledge, credentials provided by $p_{0}$, or other principals in the system, we will assume in the following discussion that the release policy for $s$ can be evaluated as satisfied or unsatisfied entirely on the basis of the truth values of quoted assertions $p_{2}$ says $e_{2}, \ldots, p_{n}$ says $e_{n}$, where $p_{2}, \ldots, p_{n}$ are willing to disclose the truth values of these assertions to $p_{0}$, but not to $p_{1}$. (A release policy that depends on information available to neither $p_{0}$ nor $p_{1}$ cannot be evaluated without the help of a third party.) We also assume that $s$ can be encoded as a short binary string of perhaps 128 bits, since this is sufficient for a symmetric key that can be used by $p_{0}$ to decrypt additional data or as proof of authorization.

Given these assumptions, $p_{1}$ can ask each $p_{i} \in\left\{p_{2}, \ldots, p_{n}\right\}$ to evaluate the corresponding expression $e_{i}$ from $p_{1}$ 's release policy. This expression may involve information that $p_{i}$ is willing to reveal to $p_{0}$, but not to $p_{1}$. In order to assert the truth of the expression $e_{i}, p_{i}$ encrypts the value 1 for $p_{0}$ 's public key using an IND-CPA-secure homomorphic encryption scheme and returns the ciphertext to $p_{1}$; otherwise, $p_{i}$ encrypts a random value and returns the resulting ciphertext. These ciphertexts reveal no information at all to $p_{1}$ because of the IND-CPA security of the encryption scheme.

Now $p_{1}$ encrypts the secret $s$ using $p_{0}$ 's public key, homomorphically combines the encryption of $s$ with each of the ciphertexts received from $p_{2}, \ldots, p_{n}$, and finally sends the combined ciphertext to $p_{0}$. If the quoted assertions $p_{2}$ says $e_{2}, \ldots, p_{n}$ says $e_{n}$ from $p_{1}$ 's release policy were all true, then $p_{0}$ receives the encryption of $s$, since homomorphically combining the encryption of $s$ with the encryption of 1 has no effect. Otherwise, one of the ciphertexts that $p_{1}$ received from $p_{2}, \ldots, p_{n}$ must have been the encryption of a random value, so $p_{0}$ receives the encryption of $s$ multiplied by a random value, which contains no more information than the random value. In either case, $p_{0}$ learns nothing about the structure of $p_{1}$ 's policy or the fact that $p_{2}, \ldots, p_{n}$ were involved in enforcing it, since $p_{0}$ always receives a single ciphertext from $p_{1}$. If $p_{0}$ fails to obtain $s$ by decrypting the ciphertext, then this fact may simply indicate that $p_{1}$ determined that the release policy for $s$ was unsatisfied based on $p_{1}$ 's local knowledge. In the following section, we describe this solution in more detail, including how $p_{2}, \ldots, p_{n}$ can enforce release policies of their own on the truth values of the assertions that they evaluate.

\section{PROTOCOL DETAILS}

Let $\mathcal{M}$ denote the message space of the IND-CPA-secure homomorphic encryption scheme, $E_{p_{i}}(m)$ denote the encryption of message $m$ using principal $p_{i}$ 's public key, and $E_{p_{i}}\left(m_{1}\right) \otimes E_{p_{i}}\left(m_{2}\right)=E_{p_{i}}\left(m_{1} \cdot m_{2}\right)$ denote the homomorphic combination of two ciphertexts encrypted using $p_{i}$ 's public key. We will assume that principals can obtain one another's public keys and that all communication takes place over secure and authenticated channels. As before, we use $p_{0}$ to denote the principal who wishes to obtain a secret $s_{1} \in \mathcal{M}$ and $p_{1}$ to denote the principal in possession of this secret.

\subsection{Core Protocol}

The secret requester $p_{0}$ first sends a message to $p_{1}$ asking for its secret $s_{1}$, along with a newly generated globally unique session identifier sid. Upon receiving this request, $p_{1}$ contacts each principal $p_{i}$ listed in its release policy for $s_{1}$ and asks it to evaluate the corresponding assertion $e_{i}$. The session identifier sid generated by $p_{0}$ is passed along with this request. At this point, $p_{1}$ may replace $s_{1}$ with a random value if $p_{1}$ decides that $p_{0}$ is not authorized to receive $s_{1}$ based on information available to $p_{1}$.

Each principal $p_{i}$ contacted by $p_{1}$ selects a local secret $s_{i}$ based on the result of evaluating $e_{i}$. If $e_{i}$ is true and $p_{i}$ is (conditionally) willing to disclose this to $p_{0}$ via $p_{1}$, then $s_{i}$ is set to 1 ; otherwise, $s_{i}$ is an element of the message space $\mathcal{M}$ chosen uniformly at random. If $p_{i}$ is willing to unconditionally reveal $s_{i}$ to $p_{0}$, then $p_{i}$ simply returns $E_{p_{0}}\left(s_{i}\right)$ to $p_{1}$. Otherwise, $p_{i}$ can make the disclosure of the truth value of $e_{i}$ contingent upon the truth of a set of quoted assertions of the same form as $p_{1}$ 's release policy for $s_{1}$ by homomorphically combining $E_{p_{0}}\left(s_{i}\right)$ with additional ciphertexts as described below. In either case, $p_{1}$ homomorphically combines the ciphertext returned by each $p_{i}$ with $E_{p_{0}}\left(s_{1}\right)$, the encryption of the secret requested by $p_{0}$, and returns the final ciphertext to $p_{0}$.

It may initially seem as though any principal $p_{i}$ contacted by $p_{1}$ can enforce its release policy for $s_{i}$ in exactly the same manner as $p_{1}$ enforces its release policy for $s_{1}$. This is almost the case, but a problem arises when the release policies of several principals create a cycle of quoted assertions in which the disclosure of each assertion depends on the disclosure of another assertion in the cycle. The purpose of the session 
identifier passed along with each request is to enable principals to detect and break such cycles.

\subsection{Policy Cycle Resolution}

The basic idea of the algorithm that we will present below is that when a requesting principal $p_{r}$ contacts a principal $p_{i}$ to ask about some assertion $e_{i}, p_{i}$ must ensure that the ciphertext that is returned to $p_{r}$ is the encryption of a random value if any quoted assertion $p_{j}$ says $e_{j}$ in $p_{i}$ 's release policy for the truth value of $e_{i}$ is false. If $p_{i}$ always waits for $p_{j}$ to return a ciphertext corresponding to the truth value of $e_{j}$ before $p_{i}$ returns an answer to $p_{r}$, however, a policy cycle will cause a deadlock.

Our solution is for $p_{i}$ to provisionally act as though the required ciphertext $c_{j}$ from $p_{j}$ was the encryption of a random value if a request for $c_{j}$ was previously sent in the current session but no reply has yet been received. That is, $p_{i}$ homomorphically combines the ciphertext $c_{i}$ that it will return to the requesting principal $p_{r}$ with the encryption of a newly generated random value. When the real response $c_{j}$ from $p_{j}$ is eventually received, a number of these random values, $r_{1}, r_{2}, \ldots, r_{n}$, may have been used, but $p_{i}$ can undo the effect of $r_{1}, r_{2}, \ldots, r_{n}$ by computing $\left(r_{1} r_{2} \cdots r_{n}\right)^{-1}$ and homomorphically combining the encryption of this quantity with $c_{j}$. Since every ciphertext encrypted for $p_{0}$ in a session is eventually combined into a single ciphertext, $\left(r_{1} r_{2} \cdots r_{n}\right)^{-1}$ will cancel out $r_{1} r_{2} \cdots r_{n}$ in the final ciphertext received by $p_{0}$.

Specifically, each principal $p_{i}$ maintains a product table indexed by the name of a principal, an assertion, and a session identifier. The product $r_{1} r_{2} \cdots r_{n}$ of all the random values that $p_{i}$ generates while waiting for an answer from $p_{j}$ concerning assertion $e_{j}$ in session sid is stored in table entry $\operatorname{product}\left[p_{j}, e_{j}, s i d\right]$ and $p_{j}$ 's answer $c_{j}$ is combined with the encryption of product $\left[p_{j}, e_{j}, s i d\right]^{-1}$ when $c_{j}$ becomes available. Upon receiving a query concerning $e_{i}$ from a requesting principal $p_{r}$ in a session initiated by $p_{0}$ and identified by sid, $p_{i}$ initializes the ciphertext $c_{i}$ that will be returned to $p_{r}$ with $E_{p_{0}}\left(s_{i}\right)$, where $s_{i}=1$ if $e_{i}$ is true and $p_{i}$ is (conditionally) willing to disclose this to $p_{0}$ via $p_{r}$, and $s_{i}$ is a freshly chosen random element of the message space $\mathcal{M}$ otherwise. Then $p_{i}$ consults its release policy for disclosing the truth value of $e_{i}$ to $p_{0}$ via principal $p_{r}$ to obtain a list of quoted assertions of the form $p_{j}$ says $e_{j}$. For each assertion $p_{j}$ says $e_{j}$ in this list, there are now two possible cases:

- If $p_{i}$ is not currently waiting for a reply from $p_{j}$ about $e_{j}$ in the current session, then $p_{i}$ queries $p_{j}$ about $e_{j}$ (also sending the session identifier sid) and assigns 1 to $\operatorname{product}\left[p_{j}, e_{j}, s i d\right]$. When $p_{j}$ eventually replies with a ciphertext $c_{j}, p_{i}$ homomorphically combines both $c_{j}$ and the encryption of product $\left[p_{j}, e_{j}, s i d\right]^{-1}$ with the ciphertext $c_{i}$ that will be returned to $p_{r}: c_{i} \leftarrow c_{i} \otimes$ $c_{j} \otimes E_{p_{0}}\left(\operatorname{product}\left[p_{j}, e_{j}, s i d\right]^{-1}\right)$.

- If $p_{i}$ has previously contacted $p_{j}$ about $e_{j}$ but has not yet received a reply (possibly because this is the second time around a cycle of quoted assertions), then $p_{i}$ chooses a random element $r$ from $\mathcal{M}$ and multiplies $\operatorname{product}\left[p_{j}, e_{j}\right.$, sid] by $r$ : $\operatorname{product}\left[p_{j}, e_{j}, s i d\right] \leftarrow$ product $\left[p_{j}, e_{j}, s i d\right] \cdot r$. Then $p_{i}$ homomorphically combines the encryption of $r$ with the ciphertext $c_{i}$ that will be returned to $p_{r}: c_{i} \leftarrow c_{i} \otimes E_{p_{0}}(r)$.

Finally, $p_{i}$ returns the resulting ciphertext $c_{i}$ to $p_{r}$.
While $p_{i}$ may be tempted to cache a ciphertext $c_{j}$ received from principal $p_{j}$ so that $p_{i}$ will never need to query $p_{j}$ more than once about any assertion within a single session, this is inadvisable because the same random value would be reused multiple times if $c_{j}$ was the encryption of a randomly chosen element of the message space. That is, reusing $c_{j}$ would not be indistinguishable from using a fresh element of the message space chosen uniformly at random.

\section{AN EXAMPLE}

Suppose that Alice is a reporter who has heard a rumor about a certain government agency. She asks Bob, who works at this agency, whether the rumor is true (this is Bob's secret $s_{B}$ ). Bob is willing to tell $s_{B}$ to Alice if his superior Carol says that it is all right (Carol says $e_{C}$ ). Carol is not willing to say that it is all right unless David also approves (David says $e_{D}$ ), while David is not willing to say that it is all right unless Carol approves (Carol says $e_{C}$ ). Suppose that Carol and David both approve of Bob telling Alice the secret, so $e_{C}$ and $e_{D}$ are true.

In our protocol, Alice first contacts Bob, who contacts Carol, which causes Carol to contact David, who then contacts Carol again. At this point the chain ends, since Carol has already contacted David concerning $e_{D}$ in the current session. The ciphertexts passed back up the chain from Carol to Alice are shown in Figure 1. At the end of the chain, Carol has not yet received a reply from David concerning $e_{D}$, so she chooses a random element $r$ from $\mathcal{M}$ and sets product $\left[\right.$ David, $e_{D}$, sid $] \leftarrow r$. The encryption of $r$ is combined with the encryption of Carol's secret $s_{C}$ (which is 1 , since $e_{C}$ is true), and the resulting ciphertext is then returned to David. At this stage Carol has revealed no information about her secret value $s_{C}$, since it is completely obscured by multiplication with the random value $r$.

Upon receiving $E_{\text {Alice }}\left(r s_{C}\right)$ from Carol, David combines it with the encryption of his secret $s_{D}$ (which is also 1 in this case, because $e_{D}$ is true) and passes the resulting ciphertext up the chain to Carol. While David does not know that this ciphertext contains the random factor $r$, he can assume that it contains the factor $s_{C}$, which would be a random value that conceals David's secret $s_{D}$ unless $e_{C}$ is true. Upon receiving the combined ciphertext from David, Carol cancels out the random factor $r$ that she added earlier by combining $E_{\text {Alice }}\left(r s_{C} s_{D}\right)$ with $E_{\text {Alice }}\left(\operatorname{product}\left[\text { David, } e_{D}, s i d\right]^{-1}\right)=$ $E_{\text {Alice }}\left(r^{-1}\right)$. Carol also multiplies the combined secret by $s_{C}$ for a second time, but this has no effect since $s_{C}=1$ (if $e_{C}$ were false, then Carol would use a new random value for $s_{C}$ each time she is queried about $e_{C}$ ). Although $r$ has now been removed from the ciphertext, Carol's secret $s_{C}$ is protected by the inclusion of the factor $s_{D}$. When Bob receives the combined ciphertext from Carol, he contributes $s_{B}$, yielding $E_{\text {Alice }}\left(r r^{-1} s_{B} s_{C}^{2} s_{D}\right)$. Since $r r^{-1}=1$ and $s_{C}^{2} s_{D}=1$, Bob's response to Alice is just $E_{\text {Alice }}\left(s_{B}\right)$. Thus Alice has no reason to believe that Bob has consulted anyone about releasing his secret value $s_{B}$.

\section{RELATED WORK}

Hidden credentials [7, 2], oblivious signature-based envelope (OSBE) [9], and multiauthority attribute-based encryption $[3,4]$ are cryptographic mechanisms that allow a message to be protected by a release policy whose satisfaction is verified by the recipient of the message. In hidden creden- 


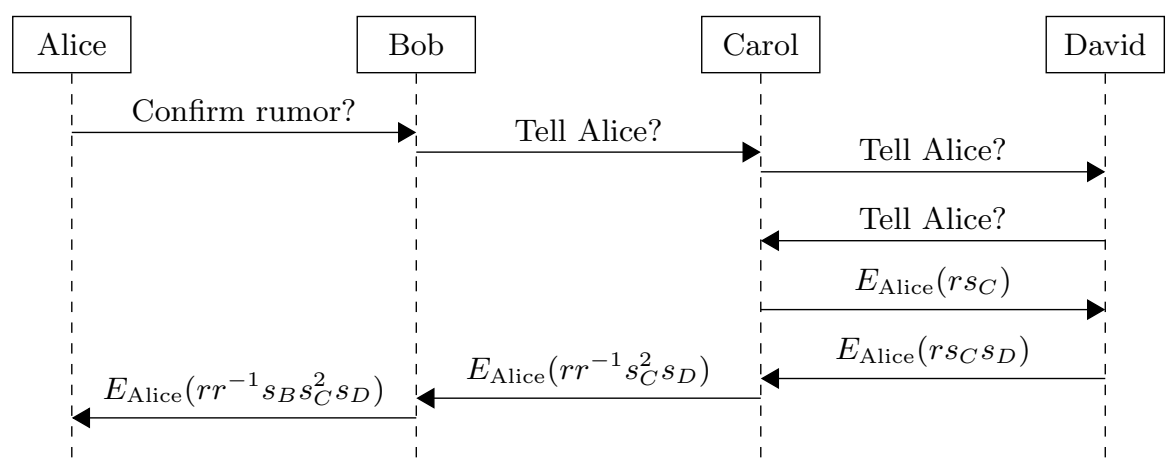

Figure 1: Queries and replies in the example of Section 4.

tials and OSBE, a message is encrypted in such a way that only a recipient who possesses certain digital credentials can decrypt the message. The identity of the intended recipient needs to be known to the message sender when this information is included in the recipient's digital credentials, as is usually the case. In multiauthority attribute-based encryption, a ciphertext is associated with a set of attributes such that any user who has been issued decryption keys (possibly by different authorities) that correspond to a satisfying set of attributes can decrypt the message. Thus no knowledge of the identities of potential recipients is needed at the time of message encryption. Unlike our work, however, in all of these schemes decrypting the message implies knowledge of at least one way of satisfying the sender's release policy.

Protocols based on scrambled circuit evaluation can be used to allow an information provider to keep a release policy partially hidden even when a message recipient satisfies the provider's policy. The three schemes of progressively greater complexity presented in [6] respectively reveal a superset of the attributes in the policy, the number of attributes in the policy that are satisfied, and an upper bound on the total number of attributes in the policy. Nevertheless, these "hidden policies with hidden credentials" protocols still require the message recipient to supply a set of credentials that potentially satisfies the unknown policy. In our system, the issuers of the credentials would be contacted directly by the information provider without any participation from the recipient; the trade-off, of course, is that partial information about the policy is revealed to the credential issuers.

Our work is also related to the notion of confidentialitypreserving distributed proof introduced in [8], which allows information providers to place release conditions that are verified by the querier on facts used in distributed inference. On the one hand, these release conditions need to be known to the querier, unlike the hidden dependencies in our protocol, but on the other, information providers communicate only with the querier and not with one another, which hides the source of dependency relationships from the providers of facts that satisfy those dependencies.

\section{CONCLUSION}

In this paper we have described a scheme for enforcing information release policies whose satisfaction cannot be verified by the principal holding the protected information, but only by the principal requesting this information. Our construction hides the information release policy being enforced from the requesting principal and at the same time hides whether the release policy was satisfied from the information provider. The quoted assertions in the information release policy can themselves have release conditions that must be satisfied and our system functions correctly even when these dependencies form cycles.

\section{Acknowledgments}

This research was supported by the National Science Foundation under grant number CCF-0916015.

\section{REFERENCES}

[1] D. Boneh. The decision Diffie-Hellman problem. In Proceedings of the Third International Symposium on Algorithmic Number Theory, pages 48-63, 1998.

[2] R. W. Bradshaw, J. E. Holt, and K. E. Seamons. Concealing complex policies with hidden credentials. In Proceedings of the 11th ACM Conference on Computer and Communications Security, pages 146-157, 2004.

[3] M. Chase. Multi-authority attribute based encryption. In Proceedings of the Fourth Theory of Cryptography Conference, pages 515-534, 2007.

[4] M. Chase and S. S. M. Chow. Improving privacy and security in multi-authority attribute-based encryption. In Proceedings of the 16th ACM Conference on Computer and Communications Security, pages 121-130, 2009.

[5] T. ElGamal. A public key cryptosystem and a signature scheme based on discrete logarithms. IEEE Transactions on Information Theory, 31(4):469-472, 1985.

[6] K. Frikken, M. Atallah, and J. Li. Attribute-based access control with hidden policies and hidden credentials. IEEE Transactions on Computers, 55(10):1259-1270, 2006.

[7] J. E. Holt, R. W. Bradshaw, K. E. Seamons, and H. Orman. Hidden credentials. In Proceedings of the 2003 ACM Workshop on Privacy in the Electronic Society, pages 1-8, 2003.

[8] A. J. Lee, K. Minami, and N. Borisov. Confidentiality-preserving distributed proofs of conjunctive queries. In Proceedings of the Fourth ACM Symposium on Information, Computer, and Communications Security, pages 287-297, 2009.

[9] N. Li, W. Du, and D. Boneh. Oblivious signature-based envelope. Distributed Computing, 17(4):293-302, 2005. 